\title{
Spotlight on measles 2010: Increased measles transmission in Ferrara, Italy, despite high vaccination coverage, March to May 2010
}

M Cova ${ }^{1}$, A Cucchi $^{2}$, G Turlà ${ }^{2}$, B Codecà ${ }^{2}, 0$ Buriani ${ }^{1}$, G Gabutti (gbtgnn@unife.it) ${ }^{2}$

1. Public Health Department, Local Health Unit, Ferrara, Italy

2. Department of Clinical and Experimental Medicine, University of Ferrara, Ferrara, Italy

We report an increase in the incidence of measles in a population with consistently high and improving immunisation coverage in Ferrara province, northern Italy. During the first six months of 2010, 19 cases were confirmed, 10 of which were hospitalised. General practitioners, paediatricians and local healthcare authorities were alerted about the outbreak and asked to notify all suspected cases. We need to further increase immunisation coverage and to maintain and implement the monitoring system.

\section{Background}

In Italy measles vaccination is recommended with a two-dose schedule, with the first dose of measlescontaining vaccine administered to children between 12 and 15 months of age and the second dose at the age of five to six years. The combined measles-mumpsrubella (MMR) vaccine was included in the national vaccination schedule in 1999, and has been provided free-of charge to all children since 2002.

Thanks to the implementation of surveillance plans and interventions to improve vaccination coverage, the incidence of measles in Italy has decreased considerably

\section{FIGURE 1}

Coverage with first dose of measles-containing vaccine by the age of 24 months in Ferrara province and EmiliaRomagna region, Italy, 1999-2008

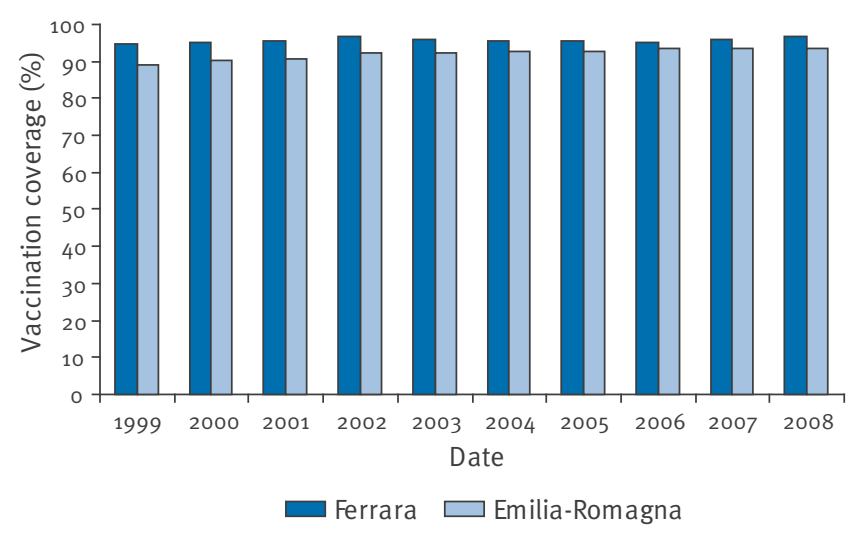

in the past decades from 150 per 100,000 0-14 year-old inhabitants in the 1960 s to 15 in 2000 and 1 in 2006 [1-2]. At the same time, the national vaccination coverage at the age of $\mathbf{2 4}$ months increased significantly from $84 \%$ in 2003 to $90 \%$ in 2006 , although with differences between regions [3]. Since 1998, the surveillance on vaccination coverage rates at national level has collected data on immunisations in newborns by a cluster sampling method. The same methodology is used to evaluate vaccination coverage rates in 16 yearold adolescents. Thus, national data on vaccination coverage for the first dose in children over two years of age and for the second dose are not available [4]. Despite recent improvements, the vaccine coverage in Italy, similar to other European countries, remains below the threshold suggested by the World Health Organization to reach elimination of the disease, i.e. country-wide at least $95 \%$ of children at the age of two years [5]. Epidemic outbreaks in several Italian regions (Piedmont, Lombardy and Emilia-Romagna) have been described in the past three years [6], an indication that measles virus is circulating. Therefore, specific preventive interventions should be strengthened. Here we describe a measles outbreak that occurred in the first

\section{FIGURE 2}

Laboratory-confirmed measles cases by sex, Ferrara province, Italy, 1 January 1999-30 June $2010(n=31)$

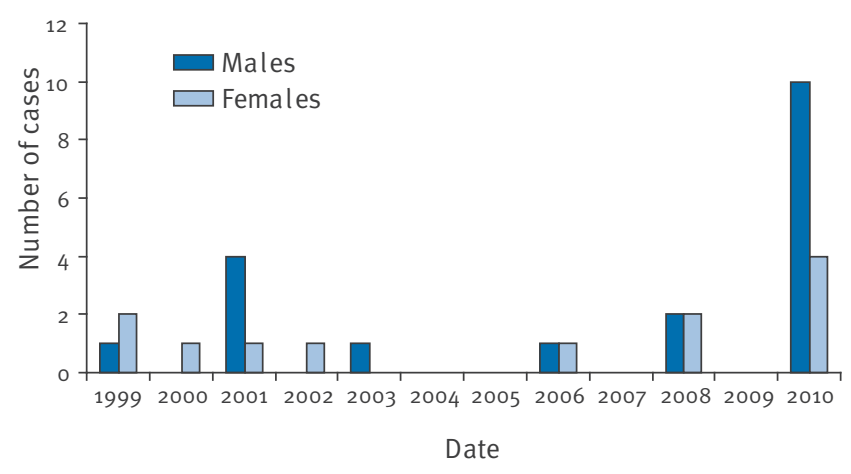


six months of 2010 in the province of Ferrara, in the Emilia-Romagna region, north-east Italy, an area with historically high immunisation coverage rates [7].

\section{Epidemiological update and outbreak description}

Ferrara had a population of approximately 360,000 inhabitants, and 2,813 newborns in 2009 [8]. According to national legislation [1] and the European case definition for measles [9], the identification of a case requires the detection of measles-specific IgM antibodies in the serum of a person notified with clinical symptoms of measles who had no record of recent vaccination. Since 1999, only notified and laboratory-confirmed (IgMpositive) cases have been reported by the healthcare workers in the local health unit to the regional information system of infectious diseases in Emilia-Romagna. Epidemiologically linked cases are notified but not reported to the information system. For each case, this database collects personal data (age, sex, place of residence), clinical information (if complications or hospitalisation occurred) and vaccination status.

In 1999 the vaccine coverage at 24 months of age was $94.9 \%$ in Ferrara province, and it has increased over the years, reaching in $200896.7 \%$ with one dose at 24 months of age and $91.1 \%$ with two doses at six years of age. The vaccine coverage has been higher in Ferrara than in the region of Emilia-Romagna as a whole since 1999 (Figure 1) [7]. Over the past ten years the measles incidence has been stable in the province and transmission has ceased spontaneously, indicating that vac- cination coverage has been high enough to break the chain of infection.

Only 17 laboratory-confirmed cases of measles were observed between 1999 and 2009, with between 0 and 5 cases per year (Figure 2). During the first six months of 2010, however, 23 cases were reported, 14 of whom were laboratory-confirmed and are shown in Figure 2. Five cases were epidemiologically linked to one of the confirmed cases but not laboratory-confirmed and hence not included in the regional information system of infectious diseases. The remaining four cases were neither laboratory-confirmed nor linked and are not further analysed here.

General practitioners, paediatricians and local healthcare authorities were alerted about the outbreak and asked to notify all cases with symptoms suggestive of measles. The following case classification was used:

- Suspected: a person with any febrile illness accompanied by rash;

- Probable: a case that met the clinical case definition [9], had non-contributory or no serological or virological test results, and was not epidemiologically linked to a confirmed case;

- Confirmed: a case that was laboratory-confirmed or that met the clinical case definition and is epidemiologically linked to a confirmed case. A laboratory-confirmed case did not need to meet the clinical case definition.

\section{TABLE}

Characteristics of measles cases, Ferrara province, Italy, 1 January-30 June 2010 (n=19)

\begin{tabular}{|c|c|c|c|c|c|c|}
\hline Case & Age & Sex & MMR vaccination status & Hospitalisation & Complication & Epidemiological link \\
\hline 1 & 16 years & $\mathrm{F}$ & Unvaccinated & No & No & No (index case) \\
\hline 2 & 48 years & $\mathrm{F}$ & Unvaccinated & Yes & Pneumonia & No \\
\hline 3 & 44 years & M & Unvaccinated & Yes & No & No \\
\hline 4 & 12 months & M & Unvaccinated & No & No & No \\
\hline 5 & 19 years & M & Unvaccinated & Yes & No & No \\
\hline 6 & 11 months & M & Unvaccinated & No & No & No \\
\hline 7 & 11 years & M & Unvaccinated & Yes & No & No (index case of the linked cluster) \\
\hline 8 & 42 years & $\mathrm{F}$ & Unvaccinated & Yes & No & No \\
\hline 9 & 11 years & $\mathrm{F}$ & Unvaccinated & No & No & Yes (primary school) \\
\hline 10 & 20 years & $M$ & Unvaccinated & Yes & No & No \\
\hline 11 & 54 years & $\mathrm{F}$ & Unvaccinated & Yes & No & No \\
\hline 12 & 49 years & M & Unvaccinated & Yes & No & No \\
\hline 13 & 10 years & M & Unvaccinated & No & No & Yes (primary school) \\
\hline 14 & 14 months & M & Unvaccinated & Yes & No & No \\
\hline 15 & 36 years & $M$ & Unvaccinated & No & No & No \\
\hline 16 & 10 years & $\mathrm{F}$ & Unvaccinated & No & No & Yes (primary school) \\
\hline 17 & 5 years & $\mathrm{F}$ & Vaccinated $^{a}$ & No & No & Yes (primary school) \\
\hline 18 & 13 months & $\mathrm{F}$ & Vaccinated $^{a}$ & No & No & Yes (sister of number 17) \\
\hline 19 & 34 years & $M$ & Unvaccinated & Yes & No & No \\
\hline
\end{tabular}

M: male; F: female;

${ }^{a}$ Vaccination with one single dose of MMR vaccine, coincidentally administered three to five days before the onset of exanthema. 
Laboratory criteria for diagnosis were a positive serologic test for measles immunoglobulin $M$ antibody, or significant rise in measles antibody level by any standard serologic assay, or isolation of measles virus from a clinical specimen [9]. A linked case was defined as a person who showed clinical signs of disease following close contact with a confirmed case during infectious period [10].

The index case, notified on 5 March, was a 16-year-old unvaccinated girl. She had no close contact with any other case nor a history of travel in areas where recent outbreaks were described. Therefore, a clear source of infection could not be identified for this case. The five epidemiologically linked cases formed a cluster notified in the period from 9 to 29 April. An 11-year-old boy was identified the index case; all cases attended the same primary school, except for a 13-month-old girl, the sister of a pupil.

The mean age among the 19 confirmed cases was 21.7 years (range 11 months to 54 years) (Table). The mean delay between the onset of the exanthema and the notification to the authorities was 3.3 days. Ten patients required hospitalisation. One patient had a complication (pneumonia). Two of the 19 cases had been vaccinated against measles with one single dose of MMR vaccine, three to five days before the onset of exanthema. Considering the incubation period for measles of 8-12 days, these two patients probably acquired the infection before immunisation and were in the incubation period at the time of vaccination [11].

\section{Control measures}

In accordance with current legislation [1], vaccination of the cases' families and other contacts has been proposed. Moreover, as a measure to control the spread of the disease, students and teachers who had no history of measles vaccination or illness were encouraged to not to attend school until there were no more cases. Recreational and work activities of each case were also recorded. General practitioners doctors, paediatricians and local healthcare authorities were requested to rapidly notify all patients with clinical symptoms suggestive of measles and to confirm the diagnosis by appropriate laboratory tests. All hospitalised patients suspected to have measles were isolated and no nosocomial transmission has been seen. No further cases were reported after 12 May. It is therefore likely that the measles outbreak has been contained through implementation of adequate control measures by the department of public health of the local healthcare unit of Ferrara.

\section{Discussion}

In 2005 the World Health Organization (WHO) had planned the elimination of measles in Europe no later than 2010 [12], but this deadline has recently been extended to 2015 [13]. In order to achieve this target, a minimum of $95 \%$ vaccination coverage with at least one dose in children at the age of two years should be reached. However, several outbreaks that occurred in recent years in Italy and other European countries are an indication that this goal has been only partially achieved. Historically, immunisation coverage in the province of Ferrara has been high, and in 1999 a vaccine coverage of $94.9 \%$ at the age of 24 months was recorded. This coverage was maintained over the following years, but the present outbreak shows how difficult it is to reduce the incidence of measles to less than one in 100,000 live births, even in an area with high vaccine coverage. It is noteworthy that the cases reported here did not give rise to large outbreaks, indicating that, together with control measures, population immunity was high enough for the outbreak to die out.

However, as long as the measles virus is imported from neighbouring areas or from other countries, a population will not be entirely without measles cases because the number of susceptible people will accumulate over time and will sustain smaller or larger outbreaks depending on how large and how concentrated the accumulated susceptible population is. Most measles outbreaks in Europe in recent years have started as a result of importation of measles from another European country, and Europe has on several occasions exported measles to measles-free areas of the world such as the Americas.

It is therefore necessary to make an extra effort to further increase immunisation coverage and to maintain and implement the monitoring system, especially in terms of quickness, completeness and accuracy of reporting.

\section{References}

1. Piano Nazionale per l'Eliminazione del Morbillo e della rosolia congenital 2003-2007. [National Plan for the elimination of measles and congenital rubella 2003-2007]. Rome: Italian Ministry of Health; 2003. Italian. Available from: www.governo. it/backoffice/allegati/20894-1712.pdf

2. Filia A, Barale A, Malaspina S, Finarelli AC, Borrini B, Moschella Let al. Focolai di morbillo in italia, gennaio 2006 - febbraio 2008. [Measles' outbreaks in Italy, January 2006 February 2008]. National Epidemiological Bulletin 2008;21(3). Italian. Available from: www.epicentro.iss.it/ben/2008/marzo/ marzo.pdf

3. ICONA Working Group. ICONA 2008: Indagine di COpertura vaccinale NAzionale nei bambini e negli adolescenti. [ICONA 2008: Survey of national vaccination coverage rate in children and adolescents]. Rome: Italian National Institute of Health; 2009. Italian. Available from: www.iss.it/binary/publ/ cont/o9_29_web.pdf

4. Filia A, Giambi C, Bella A, Ciofi degli Atti M, Declich S, Salmaso S. Sorveglianza del morbillo e della rosolia congenita e stato di avanzamento del Piano Nazionale di Eliminazione, gennaio 2009. [Measles and congenital rubella surveillance and update of the national plan for elimination]. National Epidemiological Bulletin 2009;22(2). Italian. Available from: www.epicentro.iss. it/ben/2009/febbraio/2.asp

5. Muscat M, Bang H, Wohlfahrt J, Glismann S, Mølbak K; EUVAC. NET Group. Measles in Europe: an epidemiological assessment. Lancet. 2009 Jan 31;373(9661):383-9.

6. Morbillo. [Measles]. Rome: National Institute of Health. Italian. Available from: www.epicentro.iss.it/focus/morbillo/morbillo. asp. [Accessed: 22 November 2010]

7. Coperture vaccinali nell'infanzia, 2008. [Vaccination coverage in infancy, 2008]. Bologna: Health Policy Department Emilia-Romagna Region; 2009. Italian. Available from: http://www.epicentro.iss.it/regioni/emilia/pdf/coperture vaccinali_E-R_2008.pdf 
8. Informazioni statistiche ed economiche della provincial di Ferrara 2010. [Statistics and economical update of the province of Ferrara - 2010]. Ferrara: Camera di Commercio; 2010 August. Italian. Available from: http://www.fe.camcom. it/servizi/informazione-economica/informazioni-statistiche/ Informazioni\%20Statistiche\%202010.pdf

9. Measles (Rubeola); 2010 Case Definition. Atlanta: Centers for Disease Control and Prevention; 2010 June 10. Available from: http://www.cdc.gov/ncphi/disss/nndss/casedef/ measles 2010.htm

10. Commission Decision of 28 April 2008 amending Decision 2002/253/EC laying down case definitions for reporting communicable diseases to the Community network under Decision No 2119/98/EC of the European Parliament and of the Council. 2008/426/EC. Official Journal L 159, 18/06/2008 P. 0046 - 0090. Available from: ec.europa.eu/health/ph_threats/ com/docs/1589_2008 en.pdf

11. Mason WH. Measles. In: Kliegman RM, Behrman RE, Jenson HB, Stanton BF, eds. Nelson textbook of pediatrics. 18th ed. Part XVI: Infectious diseases. Philadelphia: Saunders-Elsevier; 2007.

12. World Health Organization. WHO position on measles vaccines. Vaccine. 2009 Dec 9;27(52):7219-21.

13. World Health Organization Regional Committee for Europe. Renewed commitment to measles and rubella elimination and prevention of congenital rubella syndrome in the WHO European Region by 2015. Copenhagen, WHO Regional Office for Europe; 2010 July 23. Report EUR/RC60/15. Available from: www.euro.who.int/data/assets/pdf file/0008/119546/ RC60_edoc15.pdf 